\title{
Flow modelling of Ti6Al4V under large strains
}

\author{
HOGREFE Katharina $^{1,}$ a, BUZOLIN Ricardo ${ }^{1,2, b}$, POLETTI María Cecilia ${ }^{1,2, c}$
}

${ }^{1}$ Institute of Materials Science, Joining and Forming at Graz University of Technology. Kopernikusgasse 24/I, 8010 Graz, Austria

2Christian Doppler Laboratory for Design of High-Performance Alloys by Thermomechanical Processing

akatharina.hogrefe@tugraz.at bricardo.buzolin@tugraz.at, ccecilia.poletti@tugraz.at

Keywords: Ti64, flow modelling, hot deformation, large plastic deformation, continuous dynamic recrystallization.

\begin{abstract}
This work uses flow stresses obtained experimentally at different strain rates and temperatures to validate flow modelling results. Flow curves of Ti6A14V are measured via torsion experiments with a Gleeble ${ }^{\circledR} 3800$ up to effective strains of 8 . A physically based model that describes the evolutions of microstructure and the flow stress in the $\beta$-phase field was developed. A model of continuous dynamic recrystallization (CDRX) based on the work of Gourdet and Montheillet [1] for aluminium alloys is combined in this work with elements taken from Kocks and Mecking [2]. The model consists of a detailed description of the microstructure, based on different dislocation density populations and grain boundaries. All these internal variables evolve according to a production and a recovery term correlated mathematically with the temperature and the strain rate. The modelled output variables besides the flow stress are the total, the interior and the wall dislocation densities as well as the subgrain and grain sizes developed by continuous dynamic recrystallization. The model describes the softening occurring during large strain deformations, which is partly produced by the formation of new high angle grain boundaries (HAGB). The fraction of HAGB was used to determine the recrystallization grade, validated with microstructural characterization.
\end{abstract}

\section{Introduction}

To optimize and design hot deformation processes of the most frequently used titanium alloy Ti-6Al-4V (Ti64), the underlying metallurgical phenomena need to be described and correlated with the flow stresses evolution. This can be achieved by physically-based flow models. For the $\beta$-phase of Ti64, dynamic recovery (DRV) has been reported as the main restoration mechanism. During deformation at large strains, recrystallization processes occur in high stacking fault energy materials, namely continuous dynamic recrystallization (CDRX). [3],[4],[1] These metallurgical phenomena pose new challenges to the modelling community and need a new definition of recrystallization grade. Regarding moderate stresses a satisfying degree of predictability of the flow behaviour is reached with the existing methods. [5], [6] Contrary, for large strains only few attempts have been made to model the flow behaviour. [7], [8] This work presents the first physicallybased model based on one constitutive equation valid for all regions of strain to describe the flow behaviour and the microstructure evolution of Ti64 during hot torsion.

\section{Methodology}

Material. A commercial plate of Ti-6Al-4V was delivered after cogging treatment. The material presented primary $\alpha$ grains with a homogeneous size distribution around $10 \mu \mathrm{m}$, with a slight elongated gradient in shape in the cogging direction. Low fraction of secondary lamellar $\alpha$ and little $\beta$-phase was observed on the $\alpha$ grain boundaries. The alloy presents a $\beta$-transus temperature of $\sim 1000^{\circ} \mathrm{C}$. [9]

Hot torsion tests. Cylindrical samples with a gauge of $10 \mathrm{~mm}$ diameter and $20 \mathrm{~mm}$ length were deformed using the torsion module of a Gleeble ${ }^{\circledR} 3800$. Experiments were performed in the single $\beta$-phase field at $1030^{\circ} \mathrm{C}, 1050^{\circ} \mathrm{C}$ and $1070^{\circ} \mathrm{C}$ at three different strain rates between 0.01 and $1 \mathrm{~s}^{-1}$. The tests were conducted up to 8 revolutions, which corresponds to maximum strains of 8 . The specimens were heated up with a rate of $5 \mathrm{Ks}-1$ and held at the testing temperature for 5 minutes before the deformation started. The temperature was measured and regulated using S-thermocouples on the side of the sample. A second thermocouple on the sample's shoulder served as a reference. Immediately after hot deformation, in situ water quenching served to freeze the microstructure to avoid static phenomena at high temperatures.

Microstructure characterization. The initial and deformed samples were prepared for light optical microscopy by standard metallography methods. The samples were etched with a Kroll reagent (91 ml dist. water, $6 \mathrm{ml} \mathrm{HNO}_{3}, 3 \mathrm{ml} \mathrm{HF}$ ) for 8-15 seconds, cleaned with water and ethanol and observed with a Zeiss Axio Observer optical microscope.

Flow curves data treatment. The measured data (torque vs. torsion) was converted into flow curves (flow stress vs. strain) using the Fields and Backofen method [10]. This method uses the experimental hardening coefficient $\mathrm{n}$ and the strain rate sensitivity $\mathrm{m}$. The latter showed no dependence on the temperature and could be correlated to the experimental torsion signal by a polynomial law of second order. For the strain hardening coefficient $\mathrm{n}$, an initial value of 0.2 was set according to Lee et al. [11]. The experimental curves were fitted with an exponential function of 5 th order, followed by the calculation of the derivative of $\ln \left(\sigma_{\text {eff }}\right)$ with respect to $\ln \left(\varepsilon_{\text {eff }}\right)$, giving $n$ as a function of strain.

Flow modelling. The flow behavior of Ti64 was modelled using Matlab software for the hot deformation in the $\alpha \beta$-phase field based on the approaches proposed by Gourdet and Montheillet [1], Poletti et al. [12] and the unpublished works of Simonet-Fotso and Buzolin. The input variables of the model are the temperature, the strain rate and the average grain size of the microstructure in the $\beta$-field just before deformation. The microstructure is modelled as constituted by an average grain size, an average subgrain size, a fraction of high and low angle boundaries (LAGB and HAGB), mobile, immobile and wall dislocation densities. The modelled sequence for continuous dynamic recrystallization is illustrated in Figure 1. 


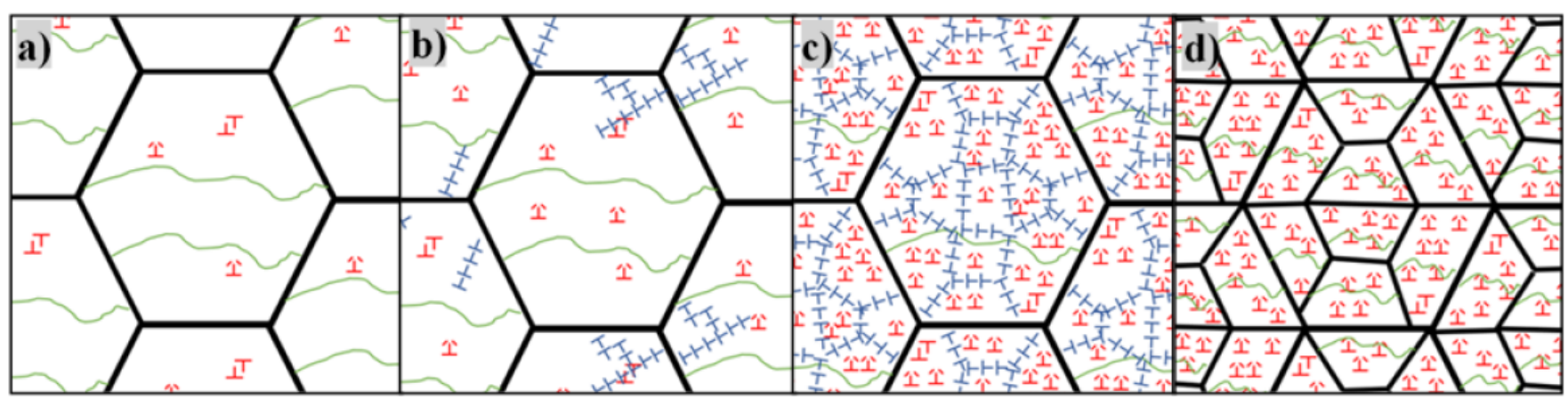

Figure 1: Schematic representation of the microstructure evolution via CDRX during hot deformation. a) Initial fully recrystallized microstructure, b) formation of LAGB by wall dislocations, c) large amount of newly formed LAGB, d) transformation of LAGB into HAGB. Immobile dislocations and dipoles are marked in red, mobile dislocations in green and wall dislocations in blue.

The average subgrain size in the starting condition was set equal to the average grain size, since the material is considered to be recrystallized. The initial value of the immobile dislocation density $\rho_{i}$ was chosen as $10^{8} \mathrm{~m} / \mathrm{m}^{3}$, and of the mobile dislocation density $\rho \mathrm{m}$ the value was $2 \cdot 10^{12} \mathrm{~m} / \mathrm{m}^{3}$. The wall dislocation density $\rho_{\mathrm{w}}$ and the fraction of LAGB are calculated according to [1]. For the latter, the lower limit of the misorientation angle $\theta_{0}$ was chosen to be $0.15^{\circ}$, and the critical misorientation angle $\theta \mathrm{c}$ as $12^{\circ}$. At this angle a LAGB is considered to convert into a HAGB.

The constitutive equation summarizes the different stress components described in the model: the thermal stress $\sigma$ th, the athermal stress $\sigma_{\text {ath }}$, and the Hall-Petch stress $\sigma_{\mathrm{HP}}$ contributing to the total flow stress according to eq. 1.

$$
\sigma=\sigma_{t h}+\sigma_{a t h}+\sigma_{H P} \quad \text { eq. } 1
$$

The Hall-Petch contribution to the stress considers the influence of both grain and subgrain sizes. The regarding shear stress contribution is calculated with the Hall-Petch constant $\mathrm{K}_{\mathrm{HAB}}$ [13] and the corresponding constant for LAGB is calculated with the critical misorientation angle $\theta \mathrm{c}$ as $\mathrm{K}_{\mathrm{LAB}}=\mathrm{K}_{\mathrm{HAB}} / \sqrt{\theta} \mathrm{c}$. The related shear stress can be derived from the Hall-Petch constants, the area fraction of the boundaries $\mathrm{S}_{-} \mathrm{V}$ and the average misorientation angle $\theta$.

$$
\begin{gathered}
\tau_{H P_{L A B}}=K_{L A B}\left(S_{V_{L A B}} \bar{\theta}\right)^{1 / 2} \\
\tau_{H P_{H A B}}=K_{L A B}\left(S_{V_{H A B}}\right)^{1 / 2} \\
\tau_{H P}=\tau_{H P_{L A B}}+\tau_{H P_{H A B}} \text { eq. } 3
\end{gathered}
$$

The shear stress is converted to flow stress via the Taylor factor $\mathrm{M}=2.53985+0.48661 \cdot \exp (-2.16688 \cdot \varepsilon)$. [15] The athermal shear stress $\tau_{\text {ath }}$ shows no dependence on temperature, and is calculated according to a Kocks-Mecking equation [2].

$$
\tau_{a t h}=\alpha \mu b\left(\rho_{i}+\rho_{m}+\rho_{w} f_{\rho w}\right)^{1 / 2}
$$

where $\mu$ is the shear modulus, $b$ is the Burger's vector and $\alpha$ is a numerical constant.

Contributions to the shear stress by the different dislocation types are shown in eq. 5. Since the strain fields produced by low energy arrangements, such as LAGB, are smaller than for isolated dislocations, the wall dislocation density contribution to the stress is added with a correction factor $\mathrm{f}_{\rho \mathrm{w}}$. The factor has a value between 0.2 and 0.7 for the investigated range of strain rates. It was fitted for a broader range of strain rates and then described by a Boltzmann function.

The thermal stress $\sigma_{-}$th is calculated from the other stress contributions including the experimentally determined yield stress $\sigma_{-}$YS .

$$
\sigma_{t h}=\sigma_{Y S}-\sigma_{a t h}-\sigma_{H P} \quad \text { eq. } 6
$$

The experimental yield stress values were fitted with a power law relationship for different strain rates and temperatures obtained by compression tests of Ti64. [14] In the deformation loop of the model, which represents the deformation process, the evolution of the microstructure and the flow stress are described using modified expression from Kocks and Mecking [2] and Gourdet and Montheillet [1].

The numerical calculation is built using incremental time steps. During hardening, dislocations can form dipoles or immobile dislocations, which is described by the hardening coefficient $\mathrm{h}_{1}$. 


$$
\frac{\partial}{\partial t}\left(\rho_{i}\right)=h_{1} \dot{\varepsilon}
$$

In terms of recovery, a recovery coefficient $\mathrm{h} \_2$ is used. Immobile dislocations and dipoles may form walls or go to already existing walls, increasing its misorientation angle. The progressive increase in misorientation leads to the formation of high angle grain boundaries. Equations 8 and 9 describe these two evolutions of dislocations, and the fractions are represented by the parameter $\alpha \_1$.

Formation of walls:

$$
\frac{\partial}{\partial t}\left(\rho_{i}\right)=h_{2} \rho_{i} \alpha_{1} \dot{\varepsilon}
$$

Moving to walls:

$$
\frac{\partial}{\partial t}\left(\rho_{i}\right)=h_{2} \rho_{i}\left(1-\alpha_{1}\right) \dot{\varepsilon}
$$

A third part describes the movement of HAGB.

$$
\frac{\partial}{\partial t}\left(\rho_{i}\right)=-\left(\rho_{i}+\rho_{m}\right) S_{V} f_{H A B} v f_{H A B}
$$

$\mathrm{S}_{\mathrm{V}}$ is the total area of boundaries per unit volume [1/m], $\mathrm{f}_{\mathrm{HAB}}$ and $\mathrm{v}_{\mathrm{HAB}}$ correspond to the surface fraction and velocity [m/s] of HAGBs. The fitting parameters $h_{1}$ and $h_{2}$ vary with temperature and strain rate, and are also a function of strain rate sensitivity $m$ and activation energy Q, as suggested by Kocks and Mecking [2].

In the described model some material specific parameters for Ti64 are incorporated, namely the Taylor factor M, the $\beta$-transus temperature, the function of the yield stress, and the Young's modulus and Poisson ratio. The last two were calculated using JMatPro ${ }^{\circledR}$ v.10. The parameters in the expression for $h_{1}$ and $h_{2}$, the constant $\alpha 1$ accounting for the recovered dislocation density forming new LAGB, the factor for the wall dislocation density $f_{\rho w}$ and a factor for the yield stress function $f_{Y S}$ are fitted using the experimental flow curves. $f_{Y S}$ was introduced as a simple multiplying factor of 0.7 , to represent the difference in yield strength between compression and torsion tests.

Figure 2 shows a scheme of the input and output variables of the CDRX model and the initialisation of the parameters. Several parameters needed to be fit with respect to the experimental flow curves and the observed microstructure. However, the fitting for all parameters except

\begin{tabular}{|c|c|c|}
\hline \multirow{4}{*}{ Input } & Value & Source \\
\hline & $4 \cdot 10^{6} \mathrm{~m}^{-1}$ & fitting \\
\hline & $1.5 \cdot 10^{7}$ & fitting \\
\hline & 0.09 & fitting \\
\hline \multirow{3}{*}{ Fitting } & 0.1089 & fitting \\
\hline & $30000 \mathrm{~J} / \mathrm{mol}$ & fitting \\
\hline & $15000 \mathrm{~J} / \mathrm{mol}$ & fitting \\
\hline \multirow[t]{2}{*}{ Literature } & function of $\varepsilon$ & reference [15] \\
\hline & 0.1 & fitting \\
\hline \multirow{3}{*}{ Experimental } & 0.0038 & fitting \\
\hline & function of $T$ & JMatPro $^{\oplus}$ simulation \\
\hline & function of $T$ & JMatPro $^{\odot}$ simulation \\
\hline \multirow{5}{*}{ Output } & function of T and $\dot{\varepsilon}$ & experimental yield stress \\
\hline & function of $\dot{\varepsilon}$ & fitting \\
\hline & $1000^{\circ} \mathrm{C}$ & reference [9] \\
\hline & function of $\dot{\varepsilon}$ & fitting \\
\hline & 8 & fitting \\
\hline
\end{tabular}
for the initial values for the hardening and recovery coefficients $h 1,0$ and $h 2,0$ was performed within a plausible range and compared to values from the literature, if available. On still open question are the values of the activation energies in the $\beta$-field. They are reported in the literature to be between 210-285 kJ/mol [8], [17]. In our present fitting $\mathrm{Q}\left(\mathrm{h}_{1}\right)$ and $\mathrm{Q}\left(\mathrm{h}_{2}\right)$ are one order of magnitude smaller.

Figure 2: Input and output variables of the proposed model for CDRX, as well as values for all used parameters.

\section{Results and discussion}

The microstructure of the samples before deformation consists mostly of globular $\alpha$, a low fraction of lamellar $\alpha$, and little $\beta$-phase. The rolling direction shows some regions with elongated grains. After deformation above the $\beta$-transus temperature the microstructure is characterized by mostly recrystallized, large grains with a martensitic structure, which is formed upon quenching, Figure 3 . The samples 
deformed in the $\beta$-field show in all cases a high amount of recrystallized grains which indicates an advanced state of recrystallization. Larger grains and a high grade of recrystallization were observed for slow strain rates, while the temperature difference of $40^{\circ} \mathrm{C}$ seems not to impact the recrystallization grade and grain size for a given strain rate. The differently deformed regions within the cylindrical torsion samples show a gradient in grain size from the sample centre to the sides, as observed in typical hot torsion experiments [16].

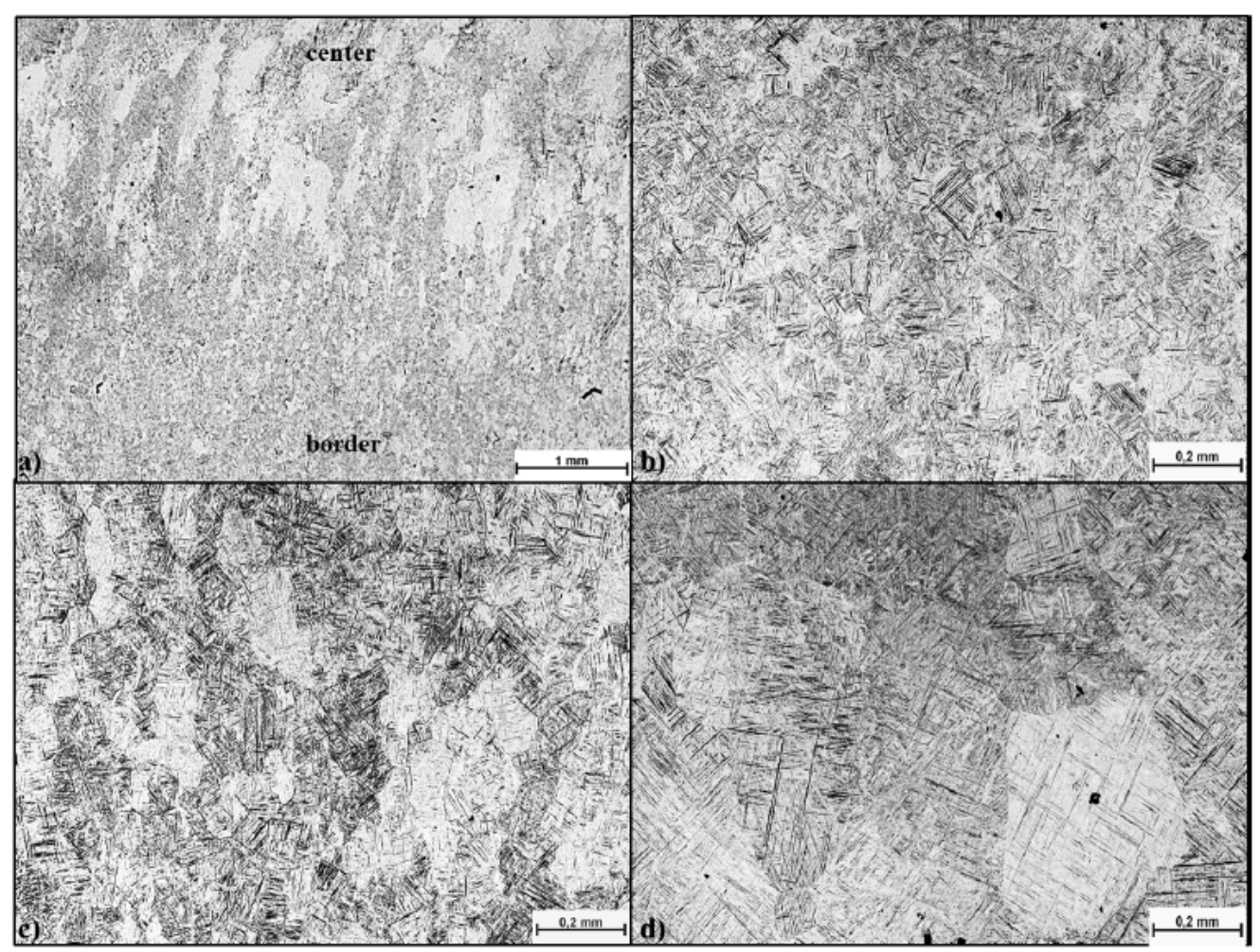

Figure 3: Microstructure of samples hot torsion at $1070{ }^{\circ} \mathrm{C}$ with a strain rate of a,b) $\left.1 \mathrm{~s}^{-1}, \mathrm{c}\right) 0.1 \mathrm{~s}^{-1}$ and d) $0.01 \mathrm{~s}^{-1}$. For the strain rate of $1 \mathrm{~s}^{-1}$, an overview of the sample is shown in a) to illustrate the grain size evolution.

Maximum flow stresses rise for low temperatures and high strain rates, as shown in Figure 5. Only the curve measured at $1070^{\circ} \mathrm{C}$ and $0.1 \mathrm{~s}^{-1}$ is an exception to this tendency and may be traced back to experimental or material irregularities. All flow curves show flow softening behaviour at higher strains, which is more pronounced for higher strain rates $\left(1 \mathrm{~s}^{-1}\right)$. At effective strains $\sim 3$ all flow curves reach a steady state. Figure 4 also shows the modelled flow curves. The model of CDRX was able to reproduce the flow stress values of Ti64 in the $\beta$-field for different strain rates and temperatures up to high strains.
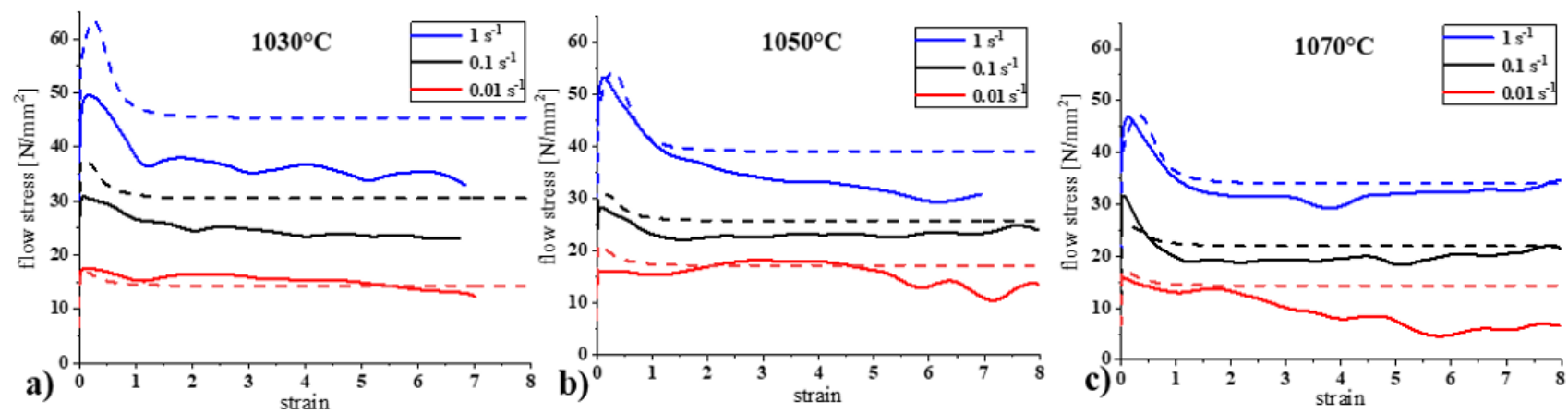

Figure 4: Modelled flow stress (dashed line) compared to experimental flow stress (solid line) for different strain rates at, a) $1030^{\circ} \mathrm{C}$, b) $1050^{\circ} \mathrm{C}$ and c) $1070^{\circ} \mathrm{C}$.

The model is able to describe the influence of strain rate and temperature on the flow stress. Both the pronounced hardening especially for high strain rates in the beginning of the flow curve and the subsequently flow softening could be modelled.

Figure 5 shows the internal variables evolution and stress contributions to the deformation at $1070^{\circ} \mathrm{C}$ and $0.01 \mathrm{~s}^{-1}$. The macroscopic total flow stress is made up to a large amount of the athermal contribution, a quarter comes from the thermal stress and Hall Petch stresses give the smallest contribution, see

Figure 5. The model also predicts the evolution of the microstructure during deformation, like the evolution of the total, wall, immobile and mobile dislocation densities. The total dislocation density (calculated as the sum of all dislocation densities) is dominated in the beginning by $\rho \mathrm{w}$, as wall dislocations form upon hardening in the beginning of deformation. For strains $\sim 0.16$, $\rho \mathrm{w}$ reaches a maximum and decreases strongly, which corresponds to the point when LAGB convert to HAGB. In the following, the total dislocation density is dominated by pi, 
which saturates at strains $\sim 1$ indicating that the formation and annihilation of immobile dislocations are in equilibrium. The contribution of mobile dislocations is minimum.
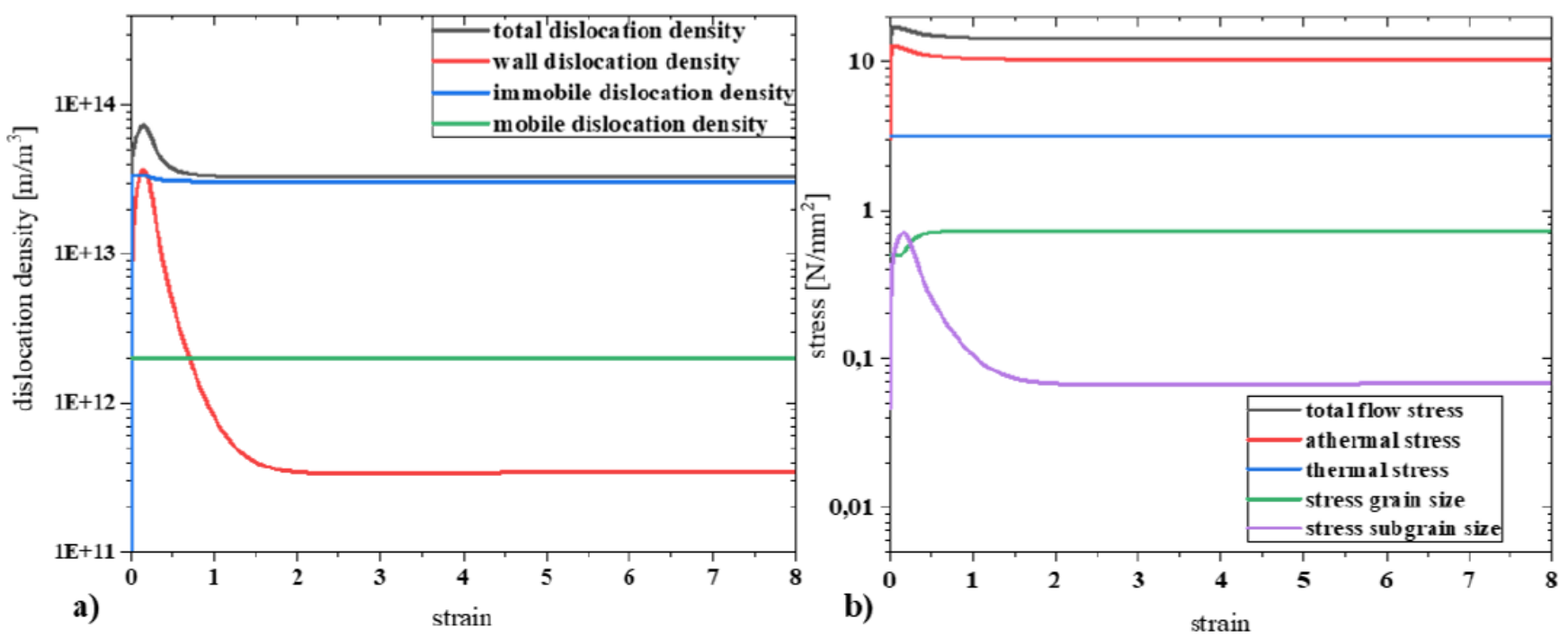

Figure 5: For a deformation at $1070^{\circ} \mathrm{C} 0.01 \mathrm{~s}^{-1}$ a) modelled evolution of different stress contributions, b) modelled evolution of different dislocation densities.

The model also delivers information about the fraction of high and low angle boundaries, and the size of grains and subgrains. The fraction of HAGBs increases with the same rate, as the fraction of LAGBs decreases. For low strain rates and high temperatures fHAGB reaches faster a value around the maximum of 1 and fLAGB a value around the minimum 0 . If the fraction of HAGB is close to 1 , a fully recrystallized microstructure is obtained. As can be seen from the micrographs from the deformed samples, the largest part of the sample is recrystallized after deformation up to strains of 8 , just as predicted by the model.

According to the model input, that assumes an initial recrystallized state, the grain size and the subgrain size start from the same initial value. While the grain size decreases and saturates at values between 114-296 $\mu \mathrm{m}$, the subgrain size reaches a minimum at strains between 0.1 and 0.3 , and saturates to values about $110-290 \mu \mathrm{m}$ for a deformation at $1070^{\circ} \mathrm{C}$ (see Figure 6). The recrystallized grain sizes for a deformation at $1070^{\circ} \mathrm{C}$ with $0.01 \mathrm{~s}^{-1}$ strain rate are in the order of few hundred $\mu \mathrm{m}$ (Figure 3) which corresponds to the model predictions.
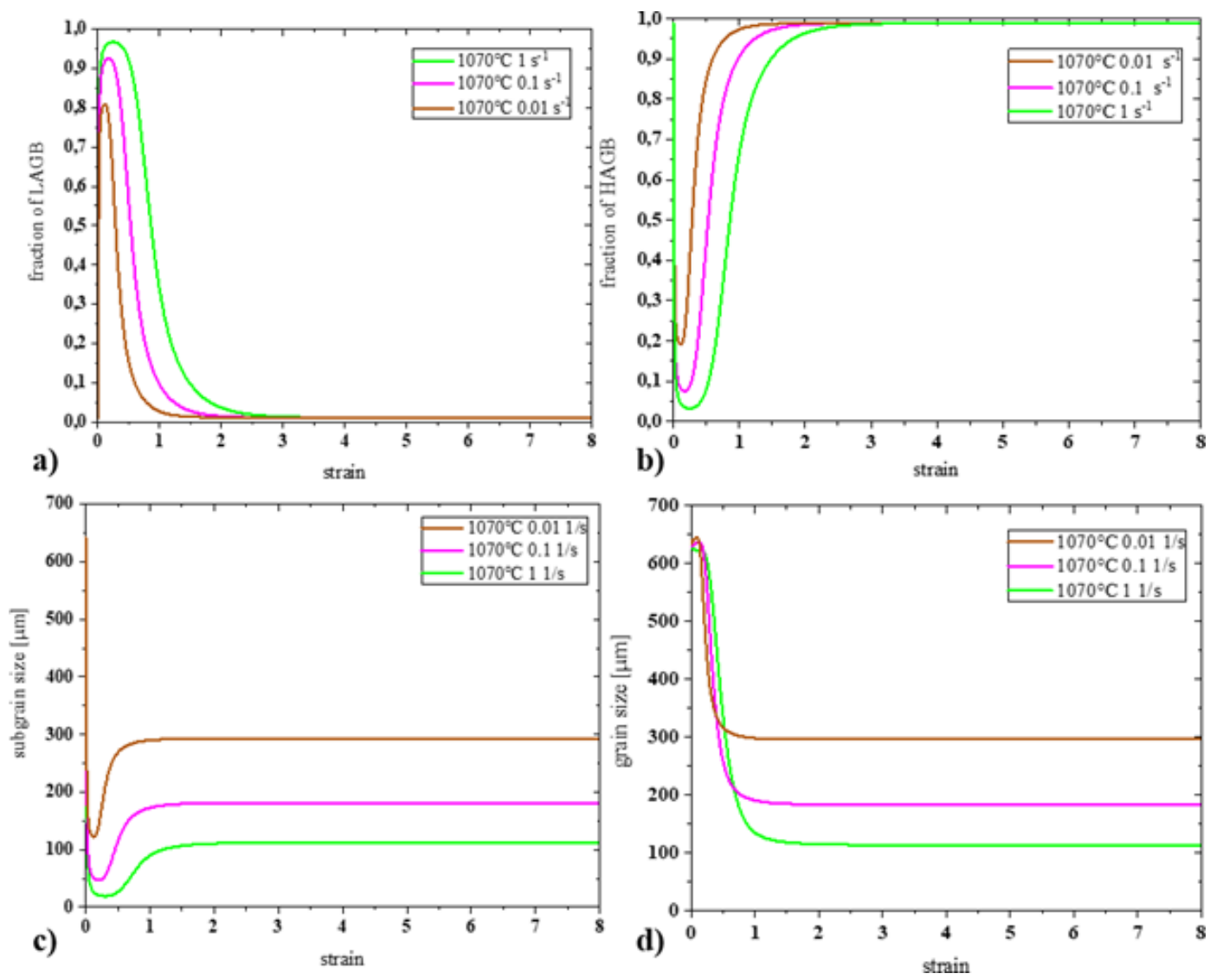
Figure 6: For a deformation at $1070^{\circ} \mathrm{C} 0.01$ 1/s a) modelled evolution of the fraction of low angle boundaries (LAGB), b) modelled evolution of the fraction of high angle boundaries (HAGB), c) modelled evolution of the subgrain size, d) modelled evolution of the grain size.

\section{$\underline{\text { Summary and conclusions }}$}

The hot deformation behaviour of Ti64 in the beta -phase field was investigated up to strains of 8 using experimental and modelling approaches. The present work may conclude the following:

CDRX is the main mechanism of deformation of Ti64 in the beta -phase field.

Hot deformation up to strains of 8 delivers fully recrystallized microstructures for the studied parameters, especially for low strain rates $\left(0.01 \mathrm{~s}^{-1}\right)$.

Recrystallization is more advanced for low strain rates and high temperatures. The progress of recrystallization is reflected in the softening of the flow curve.

Modelling of the flow curve succeeds in describing the hardening followed by a softening behaviour (formation and annihilation of wall dislocations) as well as flow stress tendencies on temperature and strain rate.

Microstructural features like the evolution of different dislocation densities, grain size and subgrain size can be modelled. Modelled grain sizes are in the range of experimentally observed ones.

Microstructural characterization of samples deformed in the beta -phase field by torsion is difficult, due to the martensite formation and heterogeneous grain sizes.

\section{$\underline{\text { Acknowledgments }}$}

The authors carried out this work under the following projects: FWF Project F37729 (Austrian Science Fund) and CD-Laboratory for Design of High-Performance Alloys by Thermomechanical Processing (Christian Doppler Society).

\section{$\underline{\text { References }}$}

[1] S. Gourdet and F. Montheillet, Acta Mater., vol. 51, no. 9, pp. 2685-2699, 2003.

[2] H. Mecking and U. F. Kocks, Acta Metall., vol. 29, no. 11, pp. 1865-1875, 1981.

[3] C. Poletti, L. Germain, F. Warchomicka, M. Dikovits, and S. Mitsche, Mater. Sci. Eng. A, vol. 651, pp. 280-290, 2016.

[4] J. Porntadawit, V. Uthaisangsuk, and P. Choungthong, Mater. Sci. Eng. A, vol. 599, pp. 212-222, 2014.

[5] X. G. Fan and H. Yang, Int. J. Plast., vol. 27, no. 11, pp. 1833-1852, 2011.

[6] R. Julien, V. Velay, V. Vidal, Y. Dahan, R. Forestier, and F. Rézaï-Aria, “Int. J. Mech. Sci., vol. 142-143, no. May, pp. 456-467, 2018.

[7] S. M. Wei Sha, First. Cambridge: Woodhead Publshing Limited, 2009.

[8] G. J. Tchein et al., " Mater. Des., vol. 161, pp. 114-123, 2019.

[9] G. Lütjering and J. C. Williams, New York: Springer-Verlag Berlin Heidelberg GmbH, 2003.

[10] J. Fields, D. S. and W. A. Backofen, ASTM Proc., vol. 57, p. 1259, 1957.

[11] W.-S. Lee and C.-F. Lin, Mater. Sci. Eng. A, vol. 241, no. 1-2, pp. 48-59, 1998.

[12] C. Poletti, R. Bureau, P. Loidolt, P. Simon, S. Mitsche, and M. Spuller, Materials (Basel)., vol. 11, no. 8, p. $1319,2018$.

[13] K. H. Chia, K. Jung, and H. Conrad, Mater. Sci. Eng. A, vol. 409, no. 1-2, pp. 32-38, 2005.

[14] L. S. Tóth, A. Molinari, and O. Bouaziz, Mater. Sci. Eng. A, vol. 524, no. 1-2, pp. 186-192, 2009.

[15] C. H. Park, J. H. Kim, Y. T. Hyun, J. T. Yeom, and N. S. Reddy, J. Alloys Compd., vol. 582, pp. 126-129, 2014.

[16] B. Eghbali and M. Shaban, J. Iron Steel Res. Int., vol. 19, no. 1, pp. 47-52, 2012.

[17] J. Luo, M. Li, H. Li, and W. Yu, Mater. Sci. Eng. A, vol. 505, no. 1-2, pp. 88-95, 2009. 